\title{
Neuroimaging in Coma, Brain Death and Related Conditions
}

Edgar Zamora, MD1; Carlos Zamora, MD, PhD²

${ }^{1}$ Montefiore Medical Center, The Bronx, NY 2University of North Carolina at Chapel Hill, Chapel Hill, NC

Purpose

Present an overview of mechanisms leading to alterations of consciousness \& describe the current role of neuroimaging.

Approach

Cases including conditions that resulted in coma and other alterations of consciousness.

Discussion

Reticular activating system (RAS):

- Neuronal network regulating arousal and consciousness.

- Tegmentum of upper pons and midbrain $\rightarrow$ thalamus/hypothalamus $\rightarrow$ cerebral cortex

Coma

- State of unarousable unresponsiveness; broad DDx-supra, infratentorial or diffuse injury.

- Clinical history and physical exam are essential but insufficient for Dx and prognosis.

- Can evolve to vegetative estate (VS) or minimally conscious state (MCS).

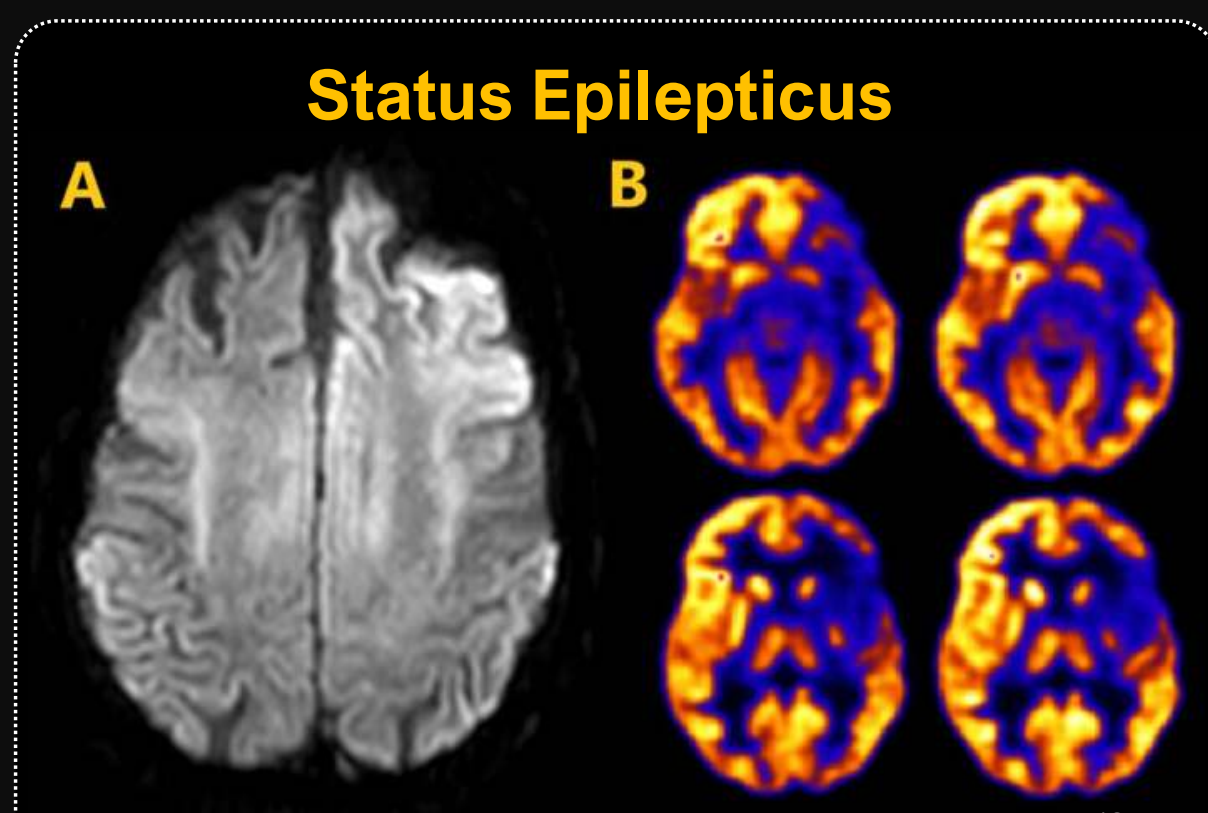

(A) DWI shows restricted diffusion in left frontal lobe. (B) ${ }^{18} \mathrm{~F}-$ FDG PET/CT: $\sqrt{ }$ perfusion in left frontal and temporal lobes.
Causes of coma are varied, largely classified into:

- Direct injuries to RAS.

- Large unilateral lesions exerting mass effect on brainstem.

- Diffuse involvement of cerebral hemispheres (e.g. toxic, drugs, metabolic, encephalitides, status epilepticus).

- Younger pts: traumatic brain injuries (TBI) \& intoxications

- Older age: metabolic disturbance, or perfusion deficits (stroke \& anoxia) are more typical.

- CT: 1st line $\rightarrow$ edema, herniation, brainstem compression. MRI: gold standard for assessing extent of injury.

Pts with midline shift $>11 \mathrm{~mm}$ usually comatose.

- Beyond structural injury $\rightarrow$ Abnormal connectivity in VS: DTI shows $\sqrt{ } \mathrm{FA}$ in WM tracts \& $\uparrow \mathrm{ADC}$ in GM/thalamus.

- Functional deficits: EEG \& evoked potentials.

- fMRI: has typically been done in subacute \& chronic stages rs fMRI: strength of default mode network connectivity has shown correlation with level of consciousness.

- ASL \& ${ }^{15} \mathrm{O}-\mathrm{H}_{2} \mathrm{O}$ PET: $\sqrt{ } \mathrm{Global}$ and regional CBF.

- ${ }^{18} \mathrm{~F}-\mathrm{FDG}-\mathrm{PET}$ : 凤 $\sqrt{ }$ global brain metabolism in VS.

Various conditions may mimic coma, including:

- Locked-in Sx: only vertical eye movement but conscious

- Akinetic mutism, psychogenic unresponsiveness.

\section{Brain Death (BD)}

- Irreversible cessation of brain function, including brainstem.

- Complete clinical examination usually sufficient but need to exclude confounders: e.g. hypothermia, intoxication., etc.

- Imaging indicated in pts with unexaminable cranial nerves, neuromuscular paralysis, heavy sedation, and others.

- Absent CBF - generally accepted as BD but may be affected by hypotension.

- Cerebral angiography best approximates gold standard but invasive and frequently impractical in highly unstable patients.

- Dx of BD in children requires 1 ancillary test for $<1$-year, and 2 ancillary tests for $<2$-month-olds; Dx cannot be made if < 37-week gestational age.

\section{Summary}

- Causes of coma are varied and can be 2ry to direct injury and/or abnormalities in functional connectivity.

- Neuroimaging is useful in determining cause and extent of injury.

- Imaging is ancillary in BD \& selected on a patient basis with special consideration in children.
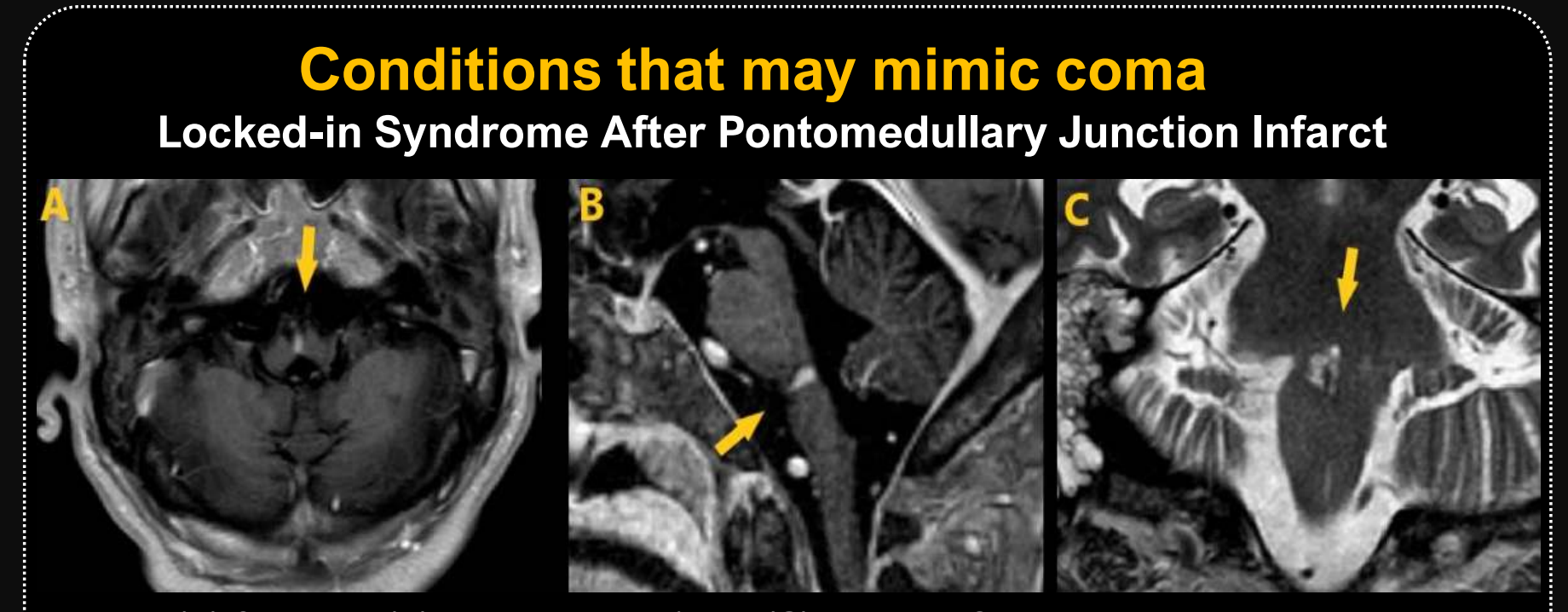

\section{Encephalopathies}

A

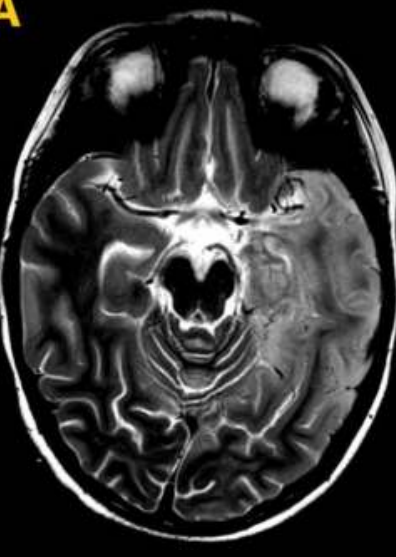

B

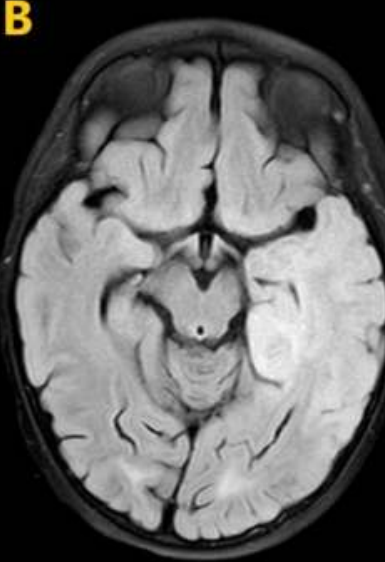

nd FLAIR (B) show left temporal

Hypoglycemic Encephalopathy Leading to Coma
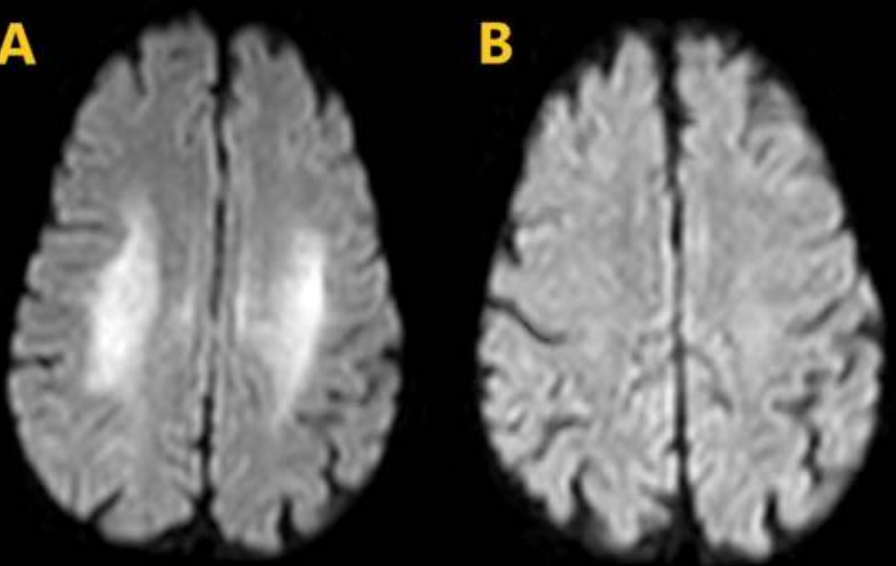

WI (A) shows restricted diffusion in corona adiata and centrum semiovale with complete esolution after 6 days of normoglycemia (B).

\section{Diffuse Cerebral Injuries Diffuse Axonal Injury}
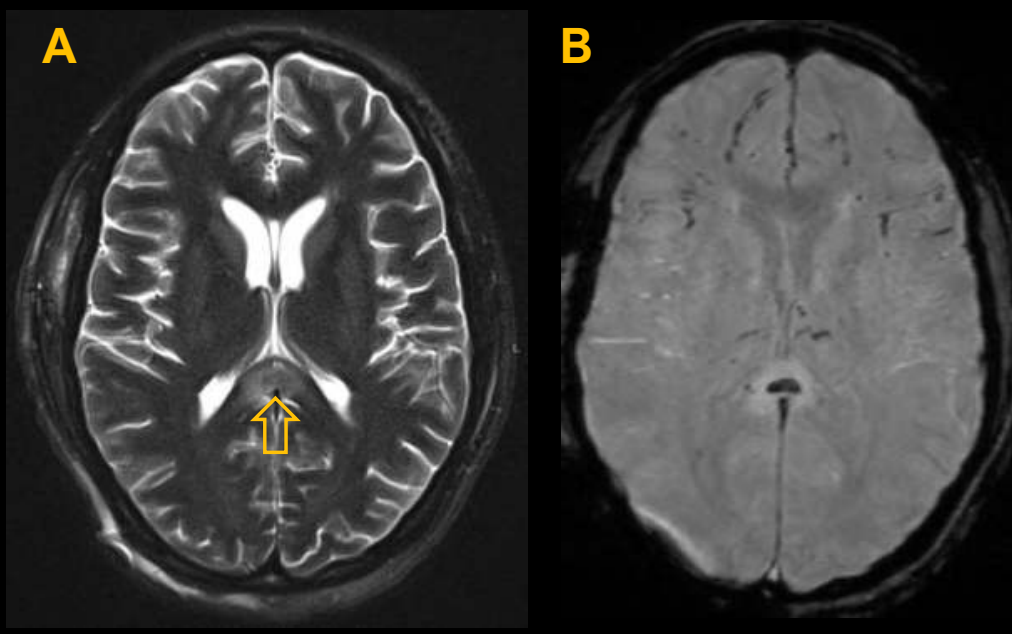

A) T2 shows callosal injury (arrow). SWI (B) shows typical linear hemorrhages along WM tracts \& thalam

Anoxic Brain Injury leading to BD

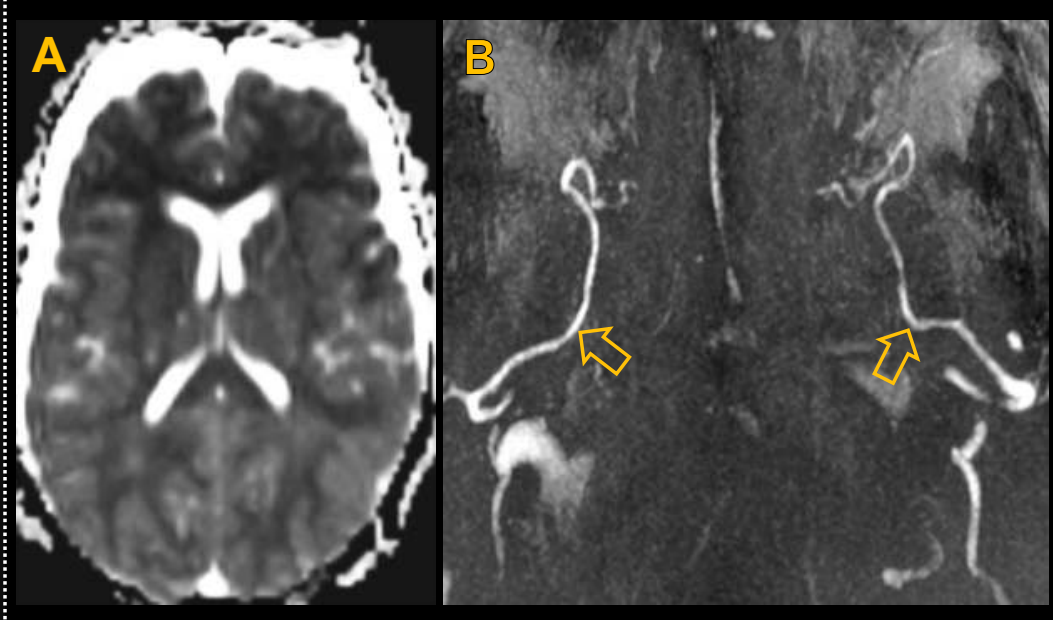

ADC map (A) shows restricted diffusion bilaterally in patient with extensive WM injury. MRA MIP (B) shows extracranial arteries but no carotids or circle of Willis 2ry to lack of intracranial perfusion. 